\title{
Generalized Algebraic Structure for Mathematical Morphology
}

\author{
Ramkumar P.B \\ Rajagir School of Engineering \& Technology \\ Rajagiri Valley \\ Kochi
}

\author{
Pramod K.V \\ Cochin University of Science \& Technology \\ Department of Computer Applications \\ Kochl
}

\begin{abstract}
Mathematical morphology is a theory of image transformations and image functional which is based on settheoretical, geometrical, and topological concepts. The methodology is particularly useful for the analysis of the geometrical structure in an image. The main goal of this paper is to introduce generalized algebraic structure for Mathematical Morphology. The following topics are discussed: introduction to mathematical morphology; generalization to algebraic structure; convex geometrical aspects of morphology. Some results presented in this paper is an extension to newly defined algebraic structure for Mathematical Morphology. . We hope that this generalization will be helpful for introducing new ideas in Morphological related works.
\end{abstract}

\section{General Terms}

Mathematical Morphology, Convex Analysis, Image Processing

\section{Keywords}

Adjunction, Morphogenetic field, Operator space ,Convex Morphological space, Image sequences ,Anchor.

\section{INTRODUCTION}

Mathematical morphology is a well-founded non-linear theory of image processing. Its geometry- oriented nature provides a framework for analyzing object shape characteristics such as size and connectivity, which are not easily accessed by linear methods.The initial form of mathematical morphology is applied to binary images. Mathematical morphology is theoretically founded on set theory. It contributes a wide range of operators to image processing, based on a few simple mathematical concepts. The operators are particularly useful for the analysis of binary images ,boundary detection, image enhancement, shape extraction, skeleton transforms and image segmentation. An image can be represented by a set of pixels. A morphological operation uses two sets of pixels, i.e., two images: the original data image to be analyzed and a structuring element (also called kernel) which is a set of pixels constituting a specific shape. A structuring element is characterized by a well- defined shape size, and origin. Its shape can be considered as a parameter to a morphological operation Mathematical morphology uses tools of algebra and operates with point sets, their connectivity and shape. Therefore, a general theory for Mathematical Morphology is a need of the day. This paper is an attempt in that direction. Without stating any fundamental results, new definitions and generalized results are given below

\section{DEFINITIONS}

\subsection{Morphogenetic field}

Let $\mathrm{X} \neq \varphi$ and $\mathrm{W} \subseteq P(X)$ such that i) $\phi, \mathrm{X} \in \mathrm{W}$, ii) If $\mathrm{B} \in \mathrm{W}$ then its complement $\bar{B} \in \mathrm{W}$ iii) If $\mathrm{B}_{\mathrm{i}} \in \mathrm{W}$ is a sequence of signals defined in $\mathrm{X}$, then $\bigcup_{n=1}^{\infty} B i \in \mathrm{W}$.

Let $A=\left\{\begin{array}{l}\phi: W \rightarrow U / \phi\left(\cup A_{i}\right)=\vee \phi\left(A_{i}\right) \\ \& \phi\left(\wedge A_{i}\right)=\wedge \phi\left(A_{i}\right)\end{array}\right\}$.

Then $W_{U}$ is called Morphogenetic field [9]where the family $W_{u}$ is the set of all image signals defined on the continuous or discrete images Plane $\mathrm{X}$ and taking values in a set $\mathrm{U}$.The pair $\left(\mathrm{W}_{\mathrm{u}}, \mathrm{A}\right)$ is called an operator space where $\mathrm{A}$ is the collection of operators defined on X.

\subsection{Morphological space}

The triplet $\left(\mathrm{X}, \mathrm{W}_{\mathrm{u}}, \mathrm{A}\right)$ consisting of a set $\mathrm{X}$, a morphogenetic field $\mathrm{W}_{\mathrm{u}}$ and an operator $\mathrm{A}$ (or collection of operators) defined on $\mathrm{X}$ is called a Morphological space[9].

\subsubsection{Discrete Morpological Spaces}

If $\mathrm{X}=\mathrm{Z}^{2}$ then it is called Discrete Morphological space

\subsubsection{Equal almost everywhere}

Let $\left(X, W_{u}, A\right)$ be a morphological space .Let

$x_{c}(\alpha), y_{c}(\alpha)$ be two functions(or signals or samples) in $\mathrm{X}$. The two functions $x_{c}(\alpha), y_{c}(\alpha)$ are equal A- almost every where if $\exists W_{1} \subseteq W_{u} \quad$ such that $\Rightarrow$ $x_{c}(\alpha)=y_{c}(\alpha) \quad \forall W_{1} \subseteq W$ and $x_{c}(\alpha), y_{c}(\alpha) \in W_{1}$.

\subsubsection{Clodum is an Operator Space}

Let $\left(X, W_{u}, A\right)$ be a morphological Let $\left(X, W_{u}, A\right)$ be a morphological space and $\left(W_{U}, A\right)$ be an operator space in $\left(X, W_{u}, A\right)$.

Let $\mathrm{X}=\mathrm{E}\left(\mathrm{E}\right.$ can be $R^{m}, Z^{m}, m=1,2, \ldots$ ) and $\mathrm{U}=\mathrm{V}$ be a complete lattice. A consists of $\vee, \wedge, *, *^{\prime}$.Then $\left(W_{U}, A\right)=$ $\left(W_{U}, \vee, \wedge, *, *^{\prime}\right)$ is called a Clodum[5] (Complete lattice ordered double monoid). 


\subsection{Non negative Space}

Let $\left(W_{-}, A\right)$ be an operator space in a morphological space $\left(X, W_{-}, A\right) \cdot L^{+}=L^{+}\left(X, W_{-}\right) \quad$ denote the collection of all non negative morphologically operator able functions (or images) on $X \rightarrow R$.

\subsection{Simple Function}

A real valued function is simple if it has only a finite number of values.

A simple operatorable function $\psi$ can be represented in the form $\quad \psi=\underset{\forall i}{\vee} a_{i} \chi_{E_{i}}$ where $\quad a_{i} \in R$ and $\psi_{E_{i}}$ is the characteristic function of a set $E_{i}$ or a structuring element in $\mathrm{X}$ in $\left(X, W_{R}, A\right)$

\subsection{Proposition}

Let $\left(X, W_{u}, A\right)$ be a morphological space and let $A_{\vee}$ be the upper slope transform. Then $A_{\vee}\left(\underset{\forall \alpha}{\vee} g_{\alpha}\right)=\underset{\forall \alpha}{\vee}\left(A_{\vee} g_{\alpha}\right), g_{\alpha} \in L^{+}$.

\subsection{Proposition}

If $\psi$ is a simple function in $L^{+}\left(X, W_{-}\right)$then the transform of $\psi$ with respect to $A_{\vee}$ is $A_{\vee}(\psi)=\underset{\forall i}{\vee} a_{i} A_{\vee}\left(E_{i}\right)$.

\subsection{Proposition}

If $\lambda$ is defined for $\mathrm{E}$ in $\mathrm{W}_{\mathrm{u}}$ by $\lambda(E)=A_{\vee}\left(\psi \chi_{E}\right)$ then $\lambda$ is a slope transform on $\mathrm{W}_{\mathrm{u}}$.

\section{TRANSLATION INVARIANCE}

\section{1 $A_{j}$ Translation invariance}

Let $\left(X, W_{u}, A\right)$ be a morphological space and $\left(W_{U}, A\right)$ be an operator space in $\left(X, W_{u}, A\right)$. An operator $A_{i} \in A$ is called $A_{j}$ translation invariant (or $T_{A_{j}}$ operator) if it commutes with any $A_{j}$ translation. i.e $A_{i} A_{j}=A_{j} A_{i} \forall j$.

\subsection{General Non Linear Super Position Principle}

Let $\left(X, W_{u}, A\right)$ be a morphological space and $\left(W_{U}, A\right)$ be an operator space in $\left(X, W_{u}, A\right)$.

An operator $B \in A$ is $A_{j}$ translation invariant iff

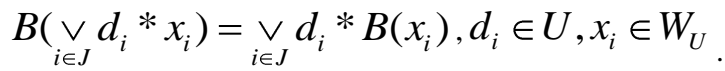

\subsubsection{Proposition}

Let $\left(X, W_{u}, A\right)$ be a morphological space and $\left(W_{U}, A\right)$ be an operator space in $\left(X, W_{u}, A\right)$.

An operator $A_{i}$ on $\left(W_{U}, A\right)$ is dilation invariant to $A_{j}$ translation iff it can be expressed as $A_{i}(F)(x)=\underset{y \in X}{\vee} F(y) * B_{y}(x)$ where $B_{y}$ is defined as $B_{y}(x)=A_{i}\left(\gamma_{y}\right)(x)$.

\subsection{2 $A_{j}$ Translation invariant system $\left(A_{j} T I\right)$}

Let $\left(X, W_{u}, A\right)$ be a morphological space and $\left(W_{U}, A\right)$ be an operator space in $\left(X, W_{u}, A\right)$.

If an operator obeys the general linear super position principle and is $A_{j}$ translation invariant then the operator defines a $A_{j}$ translation invariant system $\left(A_{j} T I\right)$.If $A_{j}$ is horizontal translation and dilation then it is called a Dilation translation invariant system (DTI)[1].Similarly Erosion translation invariant system can also be defined.

\subsubsection{Proposition}

Let $\left(X, W_{u}, A\right)$ be a morphological space and $\left(W_{U}, A\right)$ be an operator space in $\left(X, W_{u}, A\right)$.

A Signal operator $\Delta$ on $\left(W_{U}, A\right)$ is a translation invariant dilation [1] if and only if it can be represented as the supremal * convolution of the input signal with the system's response $H=\Delta(q)$.

Similarly, we can define it for erosion also.

\subsection{Slope Transforms}

\subsubsection{Proposition}

Let $\left(X, W_{u}, A\right)$ be a morphological space and $\left(W_{U}, A\right)$ be an operator space in $\left(X, W_{u}, A\right)$.

Let $A=A_{\vee}$, the upper slope transform. If $x(t) \in L^{+}$,then the upper slope transform[1] of $x(t)$ with respect to $A_{\vee}$ is $A_{\vee}(x(t))=\underset{y(t) \leq x(t)}{\operatorname{Sup}_{\vee}} A_{\downarrow}(y(t))$ where the supremum is extended over all simple functions in $L^{+}$,satisfying $0 \leq y(t) \leq x(t), \forall t \in X$.

\subsubsection{Proposition}

Let $\left(X, W_{u}, A\right)$ be a morphological space and $\left(W_{U}, A\right)$ be an operator space in $\left(X, W_{u}, A\right)$. 
If $f \in L^{+}$and $E \subseteq W$ then $f_{\chi_{E}} \in L^{+}$and the slope transform of $\mathrm{f}$ over $\mathrm{E}$ with respect to $A_{V}$ is $A_{\vee_{E}} f=A_{\vee} f_{\chi_{E}}$.

With reference to the next result [8] ,we give a lemma for slope transforms in a Morphological space.

\section{IMAGE SEQUENCES}

Let E,T be non empty sets. Denote functions from $\mathrm{E}$ into $\mathrm{T}$ by Fun(E,T).If $\mathrm{T}$ is a complete lattice, then $\operatorname{Fun}(\mathrm{E}, \mathrm{T})$ is a complete lattice too. Given an index set $\mathrm{P}$,denote set of all image sequences [8]indexed by $\mathrm{P}$, by $\mathrm{Fun}(\mathrm{E}, \mathrm{T})^{\mathrm{P}}$.Denote the elements in $\operatorname{Fun}(\mathrm{E}, \mathrm{T})^{\mathrm{P}}$ by $\mathbf{F}$ and the value of $\mathbf{F}$ at index $\mathrm{p} \in \mathrm{P}$ by $\mathrm{F}_{\mathrm{p}}$. Then $\mathbf{F} \leq \mathrm{G} \Leftrightarrow \mathrm{F}_{\mathrm{p}} \leq \mathrm{G}_{\mathrm{p}}, \mathrm{p} \in \mathrm{P} \Leftrightarrow \mathrm{F}_{\mathrm{p}}(x) \leq \mathrm{G}_{\mathrm{p}}(\mathrm{x})$, $\mathrm{p} \in \mathrm{P}, \mathrm{x} \in \mathrm{E}$.

\subsection{Lemma for Slope Transforms}

Lemma Let $\left(X, W_{u}, A\right)$ be a morphological space and $\left(W_{U}, A\right)$ be an operator space in $\left(X, W_{u}, A\right)$.

Let $A=A$ the upper slope transform. If $f, g \in L^{+}$and $f \leq g$, then

i) $A_{\vee}(f) \leq A_{\vee}(g)$ ii) If $f \in L^{+}$and $E, F \in W_{U}$ and if $E \subseteq F$ then $A_{\vee E}(f) \leq A_{\vee F}(g)$

\subsection{Fenchel conjugate}

The conjugate $x^{*}$ of $\mathrm{x}$ is defined by $x^{*}(\alpha)=\underset{v \in R^{d}}{\vee}\langle\alpha, v\rangle-x(v), \wedge(x(\alpha))=x^{*}$.The operator $\wedge$ is known as Fenchel conjugate[1].

\subsection{Proposition}

Let $\left(X, W_{u}, A\right)$ be a morphological space and $\left(W_{U}, A\right)$ be an operator space in $\left(X, W_{u}, A\right)$.

For any $x(\alpha) \in W_{U}$, the conjugate of $x(\alpha)$ is convex and lower semi continuous.

\section{CONVEX MORPHOLOGY}

\subsection{Convex Morphological Space}

Let $\left(X, W_{u}, A\right)$ be a morphological space and $\left(W_{U}, A\right)$ be an operator space in $\left(X, W_{u}, A\right)$.

If $\mathrm{X}$ is a class of concave functions then $\left(X, W_{u}, A\right)$ is called concave morphological space. If $\mathrm{X}$ is a class of convex functions then $\left(X, W_{u}, A\right)$ is called Convex morphological space.

In next proposition we give a particular property of Convex morphological space.

\subsubsection{Proposition}

If $X=k$ is a convex function and if each $k_{n}$ denote the segmented smaller linearly - sloped convex structuring components of $\mathrm{k}$ with the sizes $s_{1},\left(s_{2-}\right.$ $\left.s_{1}\right) \ldots\left(s_{n}-s_{n-1}\right)$,respectively then $\mathrm{X}$ can be written as $\mathrm{X}=k_{1} \oplus k_{2} \oplus \ldots \ldots k_{n}$.

\subsection{Anchor}

The following definition of the operator 'Anchor' can be generalized to Morphological Structures.

Given a signal $\mathrm{f}$ and an operator $\psi$, a point $\mathrm{x}$ in the domain of $\mathrm{f}$ is an anchor [8]for $\mathrm{fn}$ with respect to $\psi$ if $\psi(f)(x)=f(x) . A(f, \psi)$ is the set of anchors for $\mathrm{f}$ with respect to $\psi$ where $A(f, \psi)$ is a subset of the domain of $f$.

Anchors are useful in the computation of erosions and openings.

Let $\psi$ be an opening by $\mathrm{B}$, then the geometrical interpretation of an opening is that the lower bound of a function $\mathrm{f}$ is an anchor. If $\mathrm{f}$ has a finite domain $\mathrm{D}$ then there is at least one such global minimum, and therefore at least one anchor point. Proof of the following two results are obtained from the above argument.

\subsubsection{Theorem}

If $\gamma \mathrm{B}$ is a morphological opening on a finite domain and $\mathrm{B}$ is finite, then the set of opening anchors is always non-empty: $A(f, \gamma B) \neq 0$

\subsubsection{Theorem}

In a concave Morphological space, there exists at least one anchor point.

Proof: Let $\left(X, W_{u}, A\right)$ be a convex morphological space and $\left(W_{U}, A\right)$ be an operator space in $\left(X, W_{u}, A\right)$. Given a signal $\mathrm{f}$ belongs to $\mathrm{X}$ and an operator $\psi$ belongs to $\mathrm{A}$, a point $\mathrm{x}$ in the domain of $\mathrm{f}$ is an anchor for $\mathrm{f}$ with respect to $\psi$ if $\psi(f)(x)=f(x)$.

Let $\psi$ be an opening by B. Then the lower bound of a function $\mathrm{f}$ is an anchor. Since $\mathrm{f}$ is convex, there is at least one such global minimum, and therefore at least one anchor point.

\section{GENERALIZED ADJUNCTIONS 6.1 Definition}

Let $\left(X, W_{u}, A\right) \&\left(Y, W_{u}, \bar{A}\right)$ be morphological spaces.

The pair $(A, \bar{A})$ is called an adjunction iff $A(X) \leq Y \Leftrightarrow X \leq \bar{A}(Y)$ where $\quad \bar{A}$ is $\quad$ an inverse operator of $\mathrm{A}$. 


\subsubsection{Proposition}

Let $\left(X, W_{u}, \delta\right) \&\left(Y, W_{u}, \varepsilon\right)$ be morphological spaces with operators dilation and erosion on A. Then $\delta(X) \leq Y \Leftrightarrow X \leq \varepsilon(Y)$.

\subsubsection{Proposition (For Lattice)}

Let $\left(X, W_{u}, A\right) \&\left(Y, W_{u}, \bar{A}\right)$ be morphological spaces. The pair $(A, \bar{A})$ is called an adjunction iff $\forall u, v \in X, \exists$ an adjunction $\left(l_{u, v}, m_{v, u}\right)$ on $\mathrm{U}$ such that $\bar{A}(x(u))=\underset{v \in X}{\vee} m_{v, u}(x(v))$ and $A(y(v))=\wedge_{u \in X} l_{u, v}(y(u)), \forall u, v \in X, x, y \in W_{U}$.

\subsection{Morphological Closure}

The operator $\phi=\varepsilon \circ \delta$ defines a closure called morphological closure [7] and $\phi^{*}=\delta \circ \varepsilon$ defines a kernel, called morphological kernel.

\subsection{Separation}

Let $\left(X, W_{u}, A\right),\left(X, W_{u}, \bar{A}\right)$ be morphological spaces.

Let $(A, \bar{A})$ be adjunctions. $(X, A)$ is separated in a primary sense if $\mathrm{A}$ verifies the following two properties.

Let $x \in X, A(x) \subseteq \underset{\forall x_{i} \in W_{U}}{\cup} A\left(x_{i}\right) \Rightarrow \exists j \in I$ such $\quad$ that $A(x) \subseteq A\left(x_{j}\right), A(x)=A(y) \Rightarrow x=y \forall x, y \in X$ and $\phi=A \circ \bar{A}$ defines a morphological closure.

\section{CONCLUSION}

Mathematical morphology uses concepts from set theory, geometry and topology to analyze geometrical structures in an image. A series of operators is applied to an image in order to make certain features clearer, distinguishing meaningful information from irrelevant distortions. Therefore this attempt to generalize the theory of Mathematical Morphology is more relevant. We hope that this generalization may help researchers to find out new operators.

\section{ACKNOWLEDGMENTS}

With profound gratitude ,I express my sincere thanks to faculties of the Department of Computer Application, Cochin University of Science and Technology, Cochin, for their help and suggestions they provided. Special thanks to my colleagues at Rajagiri School of Engineering \& Technology, Cochin for their valuable suggestions.

\section{REFERENCES}

[1] Petros Maragos, Slope Transforms: Theory \&Applications to Non linear signal processing, IEEE Transactions on Image Processing. Vol .43 ,No.4,April 1995.

[2] Mathematical Morphology, John Goustias and Henk J.A.M Heijmans, I.O.S Press.

[3] H.J.A.M Heijmans, Morphological Image Operators, Boston, M.A Academic,1994 .

[4] J .Serra, Image Analysis and Mathematical Morphology, New York Academic ,1982.

[5] P .Maragos and R.W Schafer, "Morphological system for multi dimensional signal processing" Proc. IEEE,Vol,78,P.D 690-710,April 1990.

[6] Image Processing and Mathematical Morphology, Frank Y .Shih,CRC Press, 2009.

[7] Mathematical Morphology and Poset Geometry, Alain Bretto and Enzo Maria Li Marzi.

[8] Morphological operators for Image Sequences, John Goutsias, Henk J.A.M Heijmans ,K .Sivakumar

[9] Convex Geometry and Mathematical Morphology, K.V Pramod, Ramkumar P.B, communicated to 'International Journal of Computer Applications. 